\title{
Non-robustness of some impulse control problems with respect to intervention costs
}

\author{
Bernt Øksendal ${ }^{1,2}$, Jan Ubøe ${ }^{3}$, and Tusheng Zhang ${ }^{3}$ \\ ${ }^{1}$ Department of Mathematics, University of Oslo, Box 1053 Blindern, N-0316 Oslo, Norway \\ 2 Norwegian School of Economics and Business Administration, Helleveien 30, 5035 Bergen- \\ Sandviken, Norway \\ ${ }^{3}$ Stord/Haugesund College, Skåregaten 103, N-5 500, Haugesund, Norway
}

\begin{abstract}
We study how the value function (minimal cost function) $V_{c}$ of certain impulse control problems depends on the intervention $\operatorname{cost} c$. We consider the case when the cost of interfering with an impulse control of size $\zeta \in \mathbf{R}$ is given by

$$
c+|\zeta|
$$
\end{abstract}

with $c \geq 0, \lambda>0$ constants, and we show (under some assumptions) that $V_{c}$ is very sensitive (non-robust) to an increase in $c$ near $c=0$ in the sense that

\section{Introduction}

$$
\left.\frac{d V_{c}}{d c}\right|_{c=0}=+\infty
$$

A mathematical model is often a tradeoff between

i) mathematical simplicity and tractability on one hand and

ii) accuracy in the description of the real life situation that the model claims to represent, on the other.

In view of this, a natural requirement for a model to be good is robustness with respect to the parameters involved. For example, if some of the values of the parameters change slightly, this should not cause a too dramatic change in the conclusions from the model.

The purpose of this paper is to study one such robustness question in connection with a class of impulse control problems. More precisely, we study a class of impulse control problems of 1-dimensional jump diffusion processes where the cost of interfering with an impulse of size $\zeta \in \mathbf{R}$ is given by

$$
c+\lambda|\zeta|
$$

where $c \geq 0, \lambda>0$ are constants. The constant $\lambda$ is called the proportional cost coefficient and the constant $c$ is called the intervention cost. The value function/minimal cost function corresponding to $c$ when the jump diffusion starts at $y$ is denoted by $V_{c}(y)$. (See precise definitions below.) Several authors have adressed impulse control problems with a similar type of cost functional, see, e.g., [BL], [BØ2], [F], [HST], [JS], [LØ], [MØ], [MR1], [MR2], and [V].

For the particular impulse control problem to be studied below, it is well known that the mapping $c \mapsto V_{c}(y)$ is continuous at $c=0$, see [MR1]. Continuity alone, however, is not sufficient for robustness of the construction. Consider

$$
f[x]= \begin{cases}-\frac{1000}{\ln [x]} & \text { if } x>0 \\ 0 & \text { if } x=0\end{cases}
$$

Certainly, $x \mapsto f[x]$ is continuous at $x=0$. Changing $x$ from $x=0$ to $x=\frac{1}{10000}$, we change the value of $f[x]$ from 0 to more than 100. This change is in no proportion to the change in $x$. In fact, from a practical point of view it may be difficult to distinguish such a behaviour from 
a discontinuity. Therefore, to study robustness at $c=0$ it is important to study the derivative of the function at $c=0$. In this paper we prove that

$$
\left.\frac{d V_{c}(x)}{d c}\right|_{c=0}=+\infty
$$

This result can then be interpreted as follows: A small intervention cost $c>0$ will have a dramatic effect on the value function $V_{c}(y)$, in the sense that the increase in $V_{c}(y)$ is in no proportion to the increase in $c$. This phenomenon was first exhibited in [Ø2], in the case where the state process is a Brownian motion. Our paper generalizes the results to a more general class of diffusions - and even jump diffusion processes.

We now describe our setup in more detail. We want to study processes that may include jumps, so let

$$
d X_{t}=\alpha\left(X_{t}\right) d t+\beta\left(X_{t}\right) d B_{t}+h\left(X_{t-}\right) \int_{\mathbf{R}} \gamma(y) \tilde{N}(d t, d y) \quad X_{0}=x
$$

where $\tilde{N}([0, t] \times U)=N([0, t] \times U)-t m(U)$ is the compensator of the Poisson random measure $N([0, t] \times U)$ on $\mathbf{R}_{+} \times \mathbf{R}$ with the density measure $d t \times m(d y), m(d y)$ is a probability measure. We make the further assumptions that $h(x) \geq 0$ if $x \geq 0$ and that $\gamma(y) \geq 0$ everywhere. See [IW] for a discussion of these concepts. We remark that if $h=0$ or $\gamma=0$, then we are considering the classical theory without jumps.

We want to consider impulse controls $\nu=\left(\tau_{1}, \tau_{2}, \ldots ; \zeta_{1}, \zeta_{2}, \ldots\right)$ where we intervene at stopping times $\tau_{1} \leq \tau_{2} \leq \ldots$ and where we change the process by quantities $\zeta_{1}, \zeta_{2}, \ldots \in Z \subset \mathbf{R}$ at these random times ( $Z$ is a given set of admissible impulse values), i.e., that the controlled process $X_{t}^{\nu}$ satisfies

$$
X_{t}^{\nu}=x+\int_{0}^{t} \alpha\left(X_{r}^{\nu}\right) d r+\int_{0}^{t} \beta\left(X_{r}^{\nu}\right) d B_{r}+\int_{0}^{t+} \int_{\mathbf{R}} h\left(X_{r-}\right) \gamma(y) \tilde{N}(d r, d y)+\sum_{\tau_{k} \leq t} \zeta_{k}
$$

Now assume that with each intervention there is a fixed transaction cost $c>0$ and a variable $\operatorname{cost} \lambda>0$ in proportion to the size of the intervention, i.e., that the total cost of the intervention $\zeta \in Z$ is

$$
c+\lambda|\zeta|
$$

Put $Y_{t}^{\nu}=\left(s+t, X_{t}^{\nu}\right)$ when $t \geq 0$, and consider $y=(s, x)$. Let $Q^{y, v}$ be the probability law of $Y_{t}^{v}$ when $Y_{0}^{v}=y$. We assume that the system has a cost rate $f(y) \geq 0$ when the system is in the state $y$. The total expected cost $J_{c}^{\nu}(y)$ associated with a particular impulse control $\nu$, is then

$$
J_{c}^{\nu}(y)=\mathrm{E}^{y}\left[\int_{0}^{\infty} f\left(Y_{t}^{\nu}\right) d t+\sum_{k=1}^{N}\left(c+\lambda\left|\zeta_{k}\right|\right) e^{-\rho \tau_{k}}\right]
$$

where $E^{y}$ denotes expectation w.r.t. $Q^{y, v}$ and the total number $N$ of interventions may be finite or infinite. We want to find the value function

$$
V_{c}(y)=\inf _{\nu \in \mathcal{V}} J_{c}^{\nu}(y) \quad y \in \mathbf{R}^{n}
$$

where $\mathcal{V}$ is a given set of admissible impulse controls $v$, see [Ø2], and to find an optimal $\nu^{*} \in \mathcal{V}$ s.t.

$$
V_{c}(y)=J_{c}^{\nu^{*}}(y) \quad y \in \mathbf{R}^{n}
$$


In this connection the following concepts are central: From now on we will assume that $Z=$ $(-\infty, 0)$ and we define the intervention operator $\mathcal{N}: L\left(\mathbf{R}^{2}\right) \rightarrow L\left(\mathbf{R}^{2}\right)$, where $L\left(\mathbf{R}^{2}\right)$ is the space of all measurable real valued functions on $\mathbf{R}^{2}$, as follows (writing $\zeta=-\xi$ )

$$
\mathcal{N} h(y)=\mathcal{N} h(s, x)=\inf _{\xi>0}\{h(s, x-\xi)+c+\lambda \xi\}
$$

Suppose that for each $(s, x)$ there exists at least one $\xi>0$ for which the infimum in (1.7) is attained. Let $\bar{\xi}=\xi_{h}(s, x)$ be a measurable selection of such $\xi$ s. Note that if we dont have any interventions, then $Y_{t}$ is a jump diffusion process with generator $A$ which on the space $C_{0}^{2}\left(\mathbf{R}^{2}\right)$ of twice continuously differentiable functions with compact support, coincides with the integro-differential operator $L$ given by

$$
\begin{aligned}
L \phi(s, x) & =\frac{\partial \phi}{\partial s}+\alpha(x) \frac{\partial \phi}{\partial x}+\frac{1}{2} \beta^{2}(x) \frac{\partial^{2} \phi}{\partial x^{2}} \\
& +\int_{\mathbf{R}}\left(\phi(x+h(x) \gamma(y))-\phi(x)-\phi^{\prime}(x) h(x) \gamma(y)\right) m(d y)
\end{aligned}
$$

See [IW]. In particular, if $\phi(s, x)=e^{-\rho s} \psi(x)$, then we have

$$
L \phi(s, x)=e^{-\rho s} L_{0} \psi(x)
$$

where

$$
\begin{aligned}
L_{0} \psi(x):= & \frac{1}{2} \beta(x)^{2} \psi^{\prime \prime}(x)+\alpha(x) \psi^{\prime}(x)-\rho \psi(x) \\
& +\int_{\mathbf{R}}\left(\psi(x+h(x) \gamma(y))-\psi(x)-\psi^{\prime}(x) h(x) \gamma(y)\right) m(d y)
\end{aligned}
$$

In the following we will assume that we are given a family $\mathcal{V}$ of impulse controls on the form $v=\left(\tau_{1}, \tau_{2}, \ldots, \xi_{1}, \xi_{2}, \ldots\right)$, to be specified later. We assume that if $v \in \mathcal{V}$, then $Y_{t}^{v}$ exists for all $t$ a.s. (i.e., has no explosion) and

$$
\tau_{k} \rightarrow \infty \quad \text { a.s. as } k \rightarrow \infty
$$

The elements $v \in \mathcal{V}$ are called admissible impulse controls. We shall restrict ourselves to the case when the cost rate $f(s, x)$ is given by

$$
f(s, x)=e^{-\rho s} x^{2}
$$

Hence we consider

$$
J_{c}^{v}(s, x)=E^{s, x}\left[\int_{s}^{\infty} e^{-\rho t}\left(X_{t}^{v}\right)^{2} d t+\sum_{k=1}^{\infty}\left(c+\lambda \xi_{k}\right) e^{-\rho \tau_{k}}\right]
$$

when $v=\left(\tau_{1}, \tau_{2}, \ldots, \xi_{1}, \xi_{2}, \ldots\right)$. Note that with such a cost rate any negative impulse value will make matters worse if $X_{t}^{v} \leq 0$. Therefore we may assume that our family $\mathcal{V}$ of admissible controls consists only of those $v$ which - in addition to the above - makes no intervention if $X_{t}^{v} \leq 0$.

We also need the Green measure $G(z, \cdot)=G_{Y^{v}}(z, \cdot)$ of the jump diffusion $Y_{t}^{v}$, which is defined as follows

$$
G(z, F)=E^{z}\left[\int_{0}^{\infty} \mathcal{X}_{F}\left(Y_{t}^{v}\right) d t\right] ; \quad F \subset \mathbf{R}^{2} \text { Borel, } v \in \mathcal{V}
$$


In other words, $G(z, F)$ is the expected total occupation time of $Y_{t}^{v}$ in $F$ when starting from $z \in \mathbf{R}^{2}$. We will need the following results:

\section{LEMMA 1.1}

Suppose

$$
\beta\left(x_{1}\right) \neq 0 \quad \text { for some } x_{1} \in \mathbf{R}
$$

Then

$$
G\left(z, \mathbf{R} \times\left\{x_{1}\right\}\right)=0 \quad \text { for all } z \in \mathbf{R}^{2}, v \in \mathcal{V}
$$

\section{PROOF}

First we recall a well known result, see, e.g., $[\mathrm{P}]$ : If $X$ is a semimartingale and $X^{c}$ is its continuous martingale part, then for any $f \geq 0$

$$
\int_{0}^{T} f\left(X_{s}\right) d<X^{c}>_{s}=\int_{-\infty}^{\infty} L_{T}^{a}(X) f(a) d a
$$

where $\left\langle X^{c}\right\rangle_{s}$ is the quadratic variation process and $L_{T}^{a}(X)$ is the local time of the semimartingale. To prove the lemma it suffices to prove that for any $T>0$, then

$$
\int_{0}^{T} \mathcal{X}_{\mathbf{R} \times\left\{x_{1}\right\}}\left(Y_{t}^{v}\right) d t=\int_{0}^{T} \mathcal{X}_{\mathbf{R}}(s+t) \mathcal{X}_{\left\{x_{1}\right\}}\left(X_{t}^{v}\right) d t=\int_{0}^{T} \mathcal{X}_{\left\{x_{1}\right\}}\left(X_{t}^{v}\right) d t=0
$$

Since $\beta\left(x_{1}\right) \neq 0$, we have

$$
\begin{aligned}
\int_{0}^{T} \mathcal{X}_{\left\{x_{1}\right\}}\left(X_{t}^{v}\right) d t & =\beta^{-2}\left(x_{1}\right) \int_{0}^{T} \mathcal{X}_{\left\{x_{1}\right\}}\left(X_{t}^{v}\right) \beta^{2}\left(x_{1}\right) d t \\
& =\beta^{-2}\left(x_{1}\right) \int_{0}^{T} \mathcal{X}_{\left\{x_{1}\right\}}\left(X_{t}^{v}\right) \beta^{2}\left(X_{t}^{v}\right) d t \\
& =\beta^{-2}\left(x_{1}\right) \int_{0}^{T} \mathcal{X}_{\left\{x_{1}\right\}}\left(X_{t}^{v}\right) d<X^{v, c}>_{t} d t \\
& =\beta^{-2}\left(x_{1}\right) \int_{-\infty}^{\infty} L_{T}^{a}\left(X^{v}\right) \mathcal{X}_{\left\{x_{1}\right\}}(a) d a=0
\end{aligned}
$$

\section{LEMMA 1.2}

Suppose $\phi \in C^{1}\left(\mathbf{R}^{2}\right) \times C_{b}^{2}\left(\mathbf{R}^{2} \backslash\left(\mathbf{R} \times\left\{x_{1}\right\}\right)\right)$ for some $x_{1} \in \mathbf{R}$ and that the second order derivatives of $\phi$ are locally bounded near $x=x_{1}$. If $\beta\left(x_{1}\right) \neq 0$, then the generalized Dynkin formula

$$
E^{z}\left[\phi\left(Y_{\tau}^{v}\right)\right]=\phi(z)+E^{z}\left[\int_{0}^{\tau} L \phi\left(Y_{t}^{v}\right) d t\right]
$$

holds for all bounded stopping times $\tau$ which are bounded above by the exit time for $Y_{t}^{v}$ from some bounded set. 


\section{PROOF}

This follows from the classical Dynkin formula for $C^{2}$ functions, combined with the following well known approximation result: Under the above assumptions there exists a sequence $\left\{\phi_{n}\right\}_{n=1}^{\infty}$ of functions $\phi_{n} \in C^{2}\left(\mathbf{R}^{2}\right)$ such that

(i) $\phi_{n} \rightarrow \phi$ uniformly on compact subsets of $\mathbf{R}^{2}$ as $n \rightarrow \infty$

(ii) $L \phi_{n} \rightarrow L \phi$ uniformly on compact subsets of $\mathbf{R}^{2} \backslash\left(\mathbf{R} \times\left\{x_{1}\right\}\right)$ as $n \rightarrow \infty$

(iii) $L \phi_{n}$ is locally bounded near $x=x_{1}$.

A proof of (a general version of) this approximation result can, e.g., be found in [Ø1], Appendix D.

The following result is a special case of a result due to [F], Theorem III.4. It is an extension to the jump diffusion case of the verification theorem for Itô diffusions in [BØ2]. Similar types of verification principles are well known in the literature, see, e.g., [BL], and [MR2].

\section{THEOREM 1.3}

(General verification theorem)

Suppose we have found a function $\phi(s, x) \in C^{1}\left(\mathbf{R}^{2}\right)$, such that (1.12)-(1.22) hold:

$$
\text { L } \phi \text { exist a.s. } G(z, \cdot) \text { for all } z \in \mathbf{R}^{2}
$$

For all $v \in \mathcal{V}$ the following Dynkin formula holds:

$$
E^{x}\left[\phi\left(Y_{\tau}^{v}\right)\right]=\phi(y)+E^{x}\left[\int_{0}^{\tau} L \phi\left(Y_{t}^{v}\right) d t\right]
$$

for all bounded stopping times $\tau$ which are bounded above by the exit time for $Y^{v}$ from some bounded set in $\mathbf{R}^{2}$.

$$
\begin{gathered}
L \phi(y)+f(y) \geq 0 \text { a.s. } G(z, \cdot) \text { for all } z \in \mathbf{R}^{2} \\
\quad \phi \leq \mathcal{N} \phi \text { on } \mathbf{R}^{2}
\end{gathered}
$$

The family

$$
\left.\left\{\phi^{-}\left(Y_{\tau-}^{v}\right)\right\}_{\tau} \quad \text { (where } \phi^{-} \text {denotes the negative part of } \phi\right)
$$

is uniformly integrable w.r.t. $Q^{y, v}$ for all $y \in \mathbf{R}^{2}$ and all $v \in \mathcal{V}$.

$$
\phi\left(Y_{t}^{v}\right) \rightarrow 0 \text { as } t \rightarrow \infty \text {, a.s. } Q^{y, v} \text { for all }(y, v) \in \mathbf{R}^{2} \times \mathcal{V}
$$

Define

$$
D=\left\{y \in \mathbf{R}^{2} ; \phi(y)<\mathcal{N} \phi(y)\right\}
$$

\section{Suppose}

$$
L \phi(y)+f(y)=0 \text { for all } y \in D
$$


Øksendal et al.

Define the impulse control

$$
\hat{v}=\left(\hat{\tau}_{1}, \hat{\tau}_{2}, \ldots ; \hat{\xi}_{1}, \hat{\xi}_{2}, \ldots\right)
$$

inductively as follows:

Put $\hat{\tau}_{0}=0$ and then

$$
\hat{\tau}_{k+1}=\inf \left\{t>\hat{\tau}_{k} ; Y_{t}^{\hat{v}_{k}} \notin D\right\}
$$

$$
\hat{\xi}_{k+1}=\bar{\xi}\left(Y_{\hat{\tau}_{k+1}}^{\hat{v}_{k}}\right) \quad \bar{\xi} \text { is the measurable selection mentioned below (1.7)) }
$$

where $Y_{t}^{\hat{v}_{k}}$ is the result of applying the impulse control

$$
\hat{v}_{k}:=\left(\hat{\tau}_{1}, \ldots, \hat{\tau}_{k} ; \hat{\xi}_{1}, \ldots, \hat{\xi}_{k}\right)
$$

to $Y_{t}$. Suppose $\hat{v} \in \mathcal{V}$ and that

$$
\lim _{k \rightarrow \infty} E^{y}\left[\phi\left(Y_{\tau_{k}}^{\hat{v}_{k}}\right)\right]=0 \quad \text { for all } y
$$

Then

$$
\phi(y)=V_{c}(y)
$$

and $v^{*}=\hat{v}$ is an optimal impulse control.

In our situation the verification theorem can be simplified to the following:

\section{COROLLARY 1.4}

(Special verification theorem)

Suppose we can find real numbers $x_{0}, x_{1}$ with $0<x_{0}<x_{1}<\infty$ and a function $\psi \in C^{2}(\mathbf{R})$ such that

$$
L_{0} \psi(x)+x^{2}=0 \quad \text { for all } x
$$

The equation

$$
\psi^{\prime}(x)=\lambda
$$

has exactly two solutions $x=x_{0}, x=x_{1}$.

$$
\begin{gathered}
\psi\left(x_{1}\right)=\psi\left(x_{0}\right)+c+\lambda\left(x_{1}-x_{0}\right) \\
\beta\left(x_{1}\right) \neq 0 \\
-\rho\left(\psi\left(x_{1}\right)+\lambda\left(x-x_{1}\right)\right)+\alpha(x) \lambda+x^{2} \geq 0 \quad \text { for all } x>x_{1} \\
\psi^{\prime \prime}\left(x_{0}\right)>0
\end{gathered}
$$

Define

$$
\Phi(x)= \begin{cases}\psi(x) & \text { for } x<x_{1} \\ \psi\left(x_{1}\right)+\lambda\left(x-x_{1}\right) & \text { for } x \geq x_{1}\end{cases}
$$


and assume that the family

$$
\left.\left\{e^{-\rho \tau} \Phi^{-}\left(X_{\tau-}\right)\right\}_{\tau} \quad \text { (where } \Phi^{-} \text {signifies the negative part of } \Phi\right)
$$

is uniformly integrable w.r.t. $Q^{s, x}$ for all $x \in \mathbf{R}$ and that

$$
e^{-\rho t} \Phi\left(X_{t}^{v}\right) \rightarrow 0 \text { as } t \rightarrow \infty \text {, a.s. } Q^{(s, x), v} \text { for all }(s, x, v) \in \mathbf{R}^{2} \times \mathcal{V}
$$

Let

$$
\phi(s, x)=e^{-\rho s} \Phi(x)
$$

then

$$
\phi(s, x)=\inf _{v \in \mathcal{V}} E^{s, x}\left[\int_{s}^{\infty} e^{-\rho t}\left(X_{t}^{v}\right)^{2} d t+\sum_{k=1}^{N}\left(c+\lambda \xi_{k}\right) e^{-\rho \tau_{k}}\right]
$$

and the following impulse control $\hat{v}=\left(\hat{\tau}_{1}, \hat{\tau}_{2}, \ldots ; \hat{\xi}_{1}, \hat{\xi}_{2}, \ldots\right)$ is optimal:

$$
\hat{\tau}_{0}=0 \quad \text { and } \quad \hat{\tau}_{k+1}=\inf \left\{t>\hat{\tau}_{k} ; X_{t}^{\hat{v}_{k}} \geq x_{1}\right\}
$$

and

$$
\begin{gathered}
\hat{\xi}_{0}= \begin{cases}x-x_{0} & \text { if } x \geq x_{1} \\
0 & \text { otherwise }\end{cases} \\
\hat{\xi}_{k+1}=x_{1}-x_{0} \quad \text { for all } k
\end{gathered}
$$

where $X_{t}^{\hat{v}_{k}}$ is the result of applying the impulse control

$$
\hat{v}_{k}:=\left(\hat{\tau}_{1}, \ldots, \hat{\tau}_{k} ; \hat{\xi}_{1}, \ldots, \hat{\xi}_{k}\right)
$$

to $X_{t}$.

\section{PROOF}

We verify that $\phi$ satisfies all the requirements of Theorem 1.3:

First note that $\Phi$ is continuous by construction. Moreover $\Phi \in C^{1}(\mathbf{R})$ since $\psi^{\prime}\left(x_{1}\right)=\lambda$. It is also clear that $\Phi \in C^{2}\left(\mathbf{R} \backslash\left\{x_{1}\right\}\right)$. So by (1.26) and Lemma 1.2, we obtain (1.13). Moreover, $L \phi(s, x)+e^{-\rho s} x^{2}=e^{-\rho s}\left(L_{0} \Phi(x)+x^{2}\right)=0$ for $x<x_{1}$. For $x>x_{1}$ consider

$$
\int \Phi(x+h(x) \gamma(y))-\Phi(x)-\Phi^{\prime}(x) h(x) \gamma(y) m(d y)
$$

Since $0<x_{1} \leq x$, then $x+h(x) \gamma(y) \geq x_{1}$. In this set $\Phi$ is linear, and the expression in (1.37) is zero. Hence

$$
L_{0} \Phi(x)+x^{2}=-\rho\left(\psi\left(x_{1}\right)+\lambda\left(x-x_{1}\right)\right)+\alpha(x) \lambda+x^{2} \geq 0
$$

by (1.27). Hence (1.14) holds. To verify (1.15) define, for fixed $x$,

$$
h(\xi)=\psi(x-\xi)+c+\lambda \xi ; \xi \geq 0
$$


Øksendal et al.

The first order condition for a minimum of $h(\xi)$ is that

$$
0=h^{\prime}(\xi)=-\psi^{\prime}(x-\xi)+\lambda
$$

i.e.,

$$
\psi^{\prime}(x-\xi)=\lambda
$$

By (1.24) this is only possible if

$$
\xi=x-x_{0} \quad \text { or } \quad \xi=x-x_{1}
$$

provided these quantities are positive. By (1.28) we have

$$
h^{\prime \prime}\left(x-x_{0}\right)>0 \text { and } h^{\prime}(\xi)<0 \Leftrightarrow x-x_{1}<\xi<x-x_{0}
$$

so the minimum of $h(\xi)$ over $\xi \geq 0$ is attained at

$$
\xi=\hat{\xi}= \begin{cases}0 & \text { if } x \leq x_{0} \\ x-x_{0} & \text { if } x_{0}<x \leq x_{1}\end{cases}
$$

Hence

$$
\mathcal{N} \psi(x)= \begin{cases}\psi(x)+c & \text { if } x \leq x_{0} \\ \psi\left(x_{0}\right)+c+\lambda\left(x-x_{0}\right) & \text { if } x_{0}<x \leq x_{1}\end{cases}
$$

Because of (1.25) we therefore have

$$
\mathcal{N} \psi\left(x_{1}\right)=\psi\left(x_{1}\right)
$$

Moreover, if $x_{0}<x<x_{1}$, we have by (1.28)

$$
\frac{d}{d x} \mathcal{N} \psi(x)=\lambda<\psi^{\prime}(x)
$$

Therefore, by (1.39) and (1.40)

$$
\mathcal{N} \psi(x)>\psi(x) \quad \text { for } x_{0}<x<x_{1}
$$

Combining (1.38) and (1.41) we obtain

$$
\Phi(x)<\mathcal{N} \Phi(x) \quad \text { for } x<x_{1}
$$

Next, assume $x \geq x_{1}$. Then if $\xi \leq x-x_{1}$, we have

$$
\begin{aligned}
& \Phi(x-\xi)+c+\lambda \xi=\psi\left(x_{1}\right)+\lambda\left(x-\xi-x_{1}\right)+c+\lambda \xi \\
= & \psi\left(x_{1}\right)+c+\lambda\left(x-x_{1}\right)=\Phi(x)
\end{aligned}
$$

And if $\xi>x-x_{1}$, we have

$$
\begin{aligned}
& \Phi(x-\xi)+c+\lambda \xi=\psi(x-\xi)+c+\lambda \xi \\
= & \psi\left(x_{1}-\left(x_{1}-x+\xi\right)\right)+c+\lambda\left(x_{1}-x+\xi\right)+\lambda\left(x-x_{1}\right) \\
\geq & \mathcal{N} \psi\left(x_{1}\right)+\lambda\left(x-x_{1}\right)=\psi\left(x_{1}\right)+\lambda\left(x-x_{1}\right)=\Phi(x)
\end{aligned}
$$


From (1.43) and (1.44) we conclude that

$$
\mathcal{N} \Phi(x) \geq \Phi(x) \quad \text { for } x \geq x_{1}
$$

On the other hand, if we choose $\xi=x-x_{0}$, we get

$$
\Phi(x-\xi)+c+\lambda \xi=\psi\left(x_{0}\right)+c+\lambda\left(x-x_{0}\right)
$$

Hence

$$
\mathcal{N} \Phi(x)=\Phi(x) \quad \text { for } x \geq x_{1}
$$

Combining (1.42) and (1.47) we have proved (1.15). Moreover

$$
\Phi(x)<\mathcal{N} \Phi(x) \Leftrightarrow x<x_{1}
$$

To finish the proof we note that (1.16), (1.17) are direct consequences of (1.30), (1.31). (1.19) follows from (1.29) and (1.23). Finally, since $X_{\tau_{k}}^{\hat{v}_{k}}=x_{0}$, we get that

$$
\lim _{k \rightarrow \infty} E^{s, x}\left[e^{-\rho \tau_{k}} \Phi\left(X_{\tau_{k}}^{\hat{v}_{k}}\right)\right]=\lim _{k \rightarrow \infty} E^{s, x}\left[e^{-\rho \tau_{k}} \Phi\left(x_{0}\right)\right]=0
$$

by (1.10). Hence (1.22) holds and the proof of Corollary 1.4 is complete.

\section{Search strategies for candidates}

Let $x$ denote the starting point of $X_{t}$ given by (1.1) and assume that there is an interval $I_{X}=\left(x_{\text {lower }}, x_{\text {upper }}\right)$ such that the process $X_{t}$ is confined to $I_{X}$ when $x \in I_{X}$. Here $x_{\text {lower }}$ and $x_{\text {upper }}$ may be finite or infinite. Let

$$
\begin{aligned}
L \phi(s, x) & =\frac{\partial \phi}{\partial s}+\alpha(x) \frac{\partial \phi}{\partial x}+\frac{1}{2} \beta^{2}(x) \frac{\partial^{2} \phi}{\partial x^{2}} \\
& +\int_{\mathbf{R}}\left(\phi(x+h(x) \gamma(y))-\phi(x)-\phi^{\prime}(x) h(x) \gamma(y)\right) m(d y)
\end{aligned}
$$

We let $D=\left\{(s, x) \mid x_{\text {lower }}<x<x_{1}\right\}$ and we will search for a candidate $\phi(s, x)$ for the value function among functions that solve

$$
L \phi(s, x)+f(s, x)=0 \quad(s, x) \in D
$$

We restrict ourselves to the case where $f(s, x)=e^{-\rho s} x^{2}$ and search for solutions of the form $\phi(s, x)=e^{-\rho s} \psi(x)$. In this case (2.2) takes the form

$$
\begin{aligned}
L_{0} \psi(x)+x^{2}= & \frac{1}{2} \beta(x)^{2} \psi^{\prime \prime}(x)+\alpha(x) \psi^{\prime}(x)-\rho \psi(x) \\
& +\int_{\mathbf{R}}\left(\psi(x+h(x) \gamma(y))-\psi(x)-\psi^{\prime}(x) h(x) \gamma(y)\right) m(d y)+x^{2} \\
& =0
\end{aligned}
$$


Now let $\psi_{\mathrm{s}}(x)=J_{c}^{\emptyset}(0, x)$, i.e., the expected total cost when we do not intervene. Since $L_{X}=$ $L_{0}+\rho I$ is the generator of the diffusion $X_{t}^{\emptyset}, J_{c}^{\emptyset}(0, x)$ is actually $R_{\rho}(\hat{f})(x)$, where $R_{\rho}=(\rho I-$ $\left.L_{X}\right)^{-1}$ is the resolvent operator of $X_{t}^{\emptyset}$ and $\hat{f}(x)=x^{2}$. Hence

$$
\left(L_{X}-\rho I\right) J_{c}^{\emptyset}(0, \cdot)(x)=L_{0} J_{c}^{\emptyset}(0, \cdot)(x)=-x^{2}
$$

In other words, $J_{c}^{\emptyset}(0, x)$ is a special solution of $(2.3)$.

To carry out the construction to follow further below in this paper, we will need to find a solution $\psi_{\mathrm{h}}$ of the corresponding homogeneous equation such that the pair $\left(\psi_{\mathrm{s}}, \psi_{\mathrm{h}}\right)$ satisfies the following crucial properties

\section{Basic assumptions}

$\mathcal{A} 1: \lim _{x \rightarrow x_{\text {lower }}} \psi_{\mathrm{h}}^{(n)}(x)=0, n=0,1,2$

$\mathcal{A} 2: \lim _{x \rightarrow x_{\text {upper }}} \psi_{\mathrm{h}}^{(n)}(x)=+\infty, n=0,1,2$

$\mathcal{A} 3: \psi_{\mathrm{h}}^{(n)}(x)>0, x \in I_{X}, n=0,1,2$

$\mathcal{A} 4: \psi_{\mathrm{s}}^{\prime}(0) \leq 0$

$\mathcal{A} 5: \lim _{x \rightarrow x_{\text {upper }}} \frac{\psi_{\mathrm{s}}^{\prime}(x)}{\psi_{\mathrm{h}}^{\prime}(x)}=0$

$\mathcal{A} 6$ : The function $\theta(x):=\frac{\psi_{\mathrm{s}}^{\prime \prime}(x)}{\psi_{\mathrm{h}}^{\prime \prime}(x)}$ satisfies

a) $\lim _{x \rightarrow x_{\text {lower }}} \theta(x)=+\infty$

b) $\lim _{x \rightarrow x_{\text {upper }}} \theta(x)=0$

c) $\theta(x)$ is strictly decreasing with an inverse function $\theta^{-1}:[0,+\infty] \rightarrow\left[x_{\text {lower }}, x_{\text {upper }}\right]$

d) There is a point $A$ s.t.

$$
-A \psi_{h}^{\prime}\left(\theta^{-1}(A)\right)+\psi_{s}^{\prime}\left(\theta^{-1}(A)\right)-\lambda=0
$$

We remark that the above properties are satisfied in all the examples we treat in Section 3 of this paper.

We will restrict our search to functions of the form

$$
\psi_{a}(x)=-a \psi_{\mathrm{h}}(x)+\psi_{\mathrm{s}}(x)
$$

where $a>0$ is a fixed parameter to be determined. We remark that the value function $\phi(s, x)$ must satisfy

$$
0 \leq \phi(0, x) \leq \psi_{\mathrm{s}}(x)=J_{c}^{\emptyset}(0, x)
$$

In all the cases we consider later in this paper, one can easily verify that any solution of $(2.3)$ which is not of the form (2.5), will violate one or both inequalities in (2.6). Although we have no complete proof of this, we guess that this is a general principle. 


\section{PROPOSITION 2.1}

Put $\bar{x}(a)=\theta^{-1}(a)$ and let $A$ be as in (2.4). When the basic assumptions listed above are satisfied, then for each fixed $a \in(0, A)$, the equation

$$
\psi_{a}^{\prime}(x)=\lambda
$$

has exactly two solutions $x_{0}(a)$ and $x_{1}(a)$ s.t. $0<x_{0}(a)<\bar{x}(a)<x_{1}(a)$. Moreover

$$
\lim _{a \rightarrow A-} x_{0}(a)=\lim _{a \rightarrow A-} x_{1}(a)=\bar{x}(A)
$$

\section{PROOF}

For each $a \in(0,+\infty)$ put $f_{a}(x)=\psi_{a}^{\prime}(x)-\lambda=-a \psi_{\mathrm{h}}^{\prime}(x)+\psi_{\mathrm{s}}^{\prime}(x)-\lambda$. Then

$$
f_{a}^{\prime}(x)=-a \psi_{\mathrm{h}}^{\prime \prime}(x)+\psi_{\mathrm{s}}^{\prime \prime}(x)=\psi_{\mathrm{h}}^{\prime \prime}(x)(\theta(x)-a)
$$

Hence since $\psi_{\mathrm{h}}^{\prime \prime}(x)>0, f_{a}^{\prime}(x)=0 \Leftrightarrow x=\bar{x}(a)$. It is easy to see that if $x<\bar{x}(a)$, then $f_{a}^{\prime}(x)>0$ and if $x>\bar{x}(a)$, then $f_{a}^{\prime}(x)<0$. Next observe that by $\mathcal{A} 4$ and $\mathcal{A} 5$

$$
f_{a}(0)<0 \quad \text { and also } \quad \lim _{x \rightarrow x_{\text {upper }}} f_{a}(x)<0
$$

Now consider $h(a):=f_{a}(\bar{x}(a))$. Then

$$
\frac{d}{d a} h(a)=-\psi_{h}^{\prime}(\bar{x}(a))+f_{a}^{\prime}(\bar{x}(a)) \frac{d}{d a} \bar{x}(a)=-\psi_{h}^{\prime}(\bar{x}(a))<0
$$

By assumption $\mathcal{A} 6 \mathrm{~d}), h(A)=0$. Hence $h(a)>0 \Leftrightarrow a \in(0, A)$. So for all $a \in(0, A)$, $f_{a}(\bar{x}(a))>0$. Combining this with $(2.10)$ we see that the equation $f_{a}(x)=0$ has exactly two solutions $x_{0}(a)$ and $x_{1}(a)$ s.t. $x_{0}(a)<\bar{x}(a)<x_{1}(a)$. Moreover, if we differentiate the equation $f_{a}(x)=0$ w.r.t. $a$, we get

$$
\frac{d}{d a} x_{0}(a)=\frac{\psi_{\mathrm{h}}^{\prime}\left(x_{0}(a)\right)}{f_{a}^{\prime}\left(x_{0}(a)\right)}>0 \quad \text { and } \quad \frac{d}{d a} x_{1}(a)=\frac{\psi_{\mathrm{h}}^{\prime}\left(x_{1}(a)\right)}{f_{a}^{\prime}\left(x_{1}(a)\right)}<0
$$

Hence the limits $\lim _{a \rightarrow A-} x_{0}(a)=\hat{x}_{0}$ and $\lim _{a \rightarrow A-} x_{1}(a)=\hat{x}_{1}$ exist. Since both limits must satisfy the equation $f_{A}(x)=0$, which is satisfied if and only if $x=\bar{x}(A)$, this completes the proof of the proposition.

\section{LEMMA 2.2}

For each $a \in(0, A)$ let $x_{0}=x_{0}(a)$ and $x_{1}=x_{1}(a)$ be the two solutions of $\psi_{a}^{\prime}(x)=\lambda$ given by Proposition 2.1. Put

$$
g(a):=\psi_{a}\left(x_{1}(a)\right)-\psi_{a}\left(x_{0}(a)\right)-\lambda\left(x_{1}(a)-x_{0}(a)\right)
$$

Then

$$
\frac{d}{d a} g(a)=\psi_{\mathrm{h}}\left(x_{0}(a)\right)-\psi_{\mathrm{h}}\left(x_{1}(a)\right)<0
$$


Moreover

$$
\lim _{a \rightarrow A-} g(a)=0 \quad \text { and } \quad \lim _{a \rightarrow 0+} g(a)=L>0
$$

We may have $L=+\infty$.

PROOF

$$
g(a)=-a \psi_{\mathrm{h}}\left(x_{1}\right)+\psi_{\mathbf{s}}\left(x_{1}\right)+a \psi_{\mathrm{h}}\left(x_{0}\right)-\psi_{\mathbf{s}}\left(x_{0}\right)-\lambda\left(x_{1}-x_{0}\right)
$$

In this proof we let ' denote differentiation w.r.t. $a$. Then we get

$$
\begin{aligned}
g^{\prime}(a) & =-\psi_{\mathrm{h}}\left(x_{1}\right)-a \psi_{\mathrm{h}}^{\prime}\left(x_{1}\right) x_{1}^{\prime}+\psi_{\mathrm{s}}^{\prime}\left(x_{1}\right) x_{1}^{\prime} \\
& +\psi_{\mathrm{h}}\left(x_{0}\right)+a \psi_{\mathrm{h}}^{\prime}\left(x_{0}\right) x_{0}^{\prime}-\psi_{\mathrm{s}}^{\prime}\left(x_{0}\right) x_{0}^{\prime}-\lambda\left(x_{1}^{\prime}-x_{0}^{\prime}\right) \\
& =\psi_{\mathrm{h}}\left(x_{0}\right)-\psi_{\mathrm{h}}\left(x_{1}\right)+\left(-a \psi_{\mathrm{h}}^{\prime}\left(x_{1}\right)+\psi_{\mathrm{s}}^{\prime}\left(x_{1}\right)-\lambda\right) x_{1}^{\prime} \\
& -\left(-a \psi_{\mathrm{h}}^{\prime}\left(x_{0}\right)+\psi_{\mathrm{s}}^{\prime}\left(x_{0}\right)-\lambda\right) x_{0}^{\prime} \\
& =\psi_{\mathrm{h}}\left(x_{0}\right)-\psi_{\mathrm{h}}\left(x_{1}\right)+\left(\psi_{a}^{\prime}\left(x_{1}\right)-\lambda\right) x_{1}^{\prime}-\left(\psi_{a}^{\prime}\left(x_{0}\right)-\lambda\right) x_{0}^{\prime} \\
& =\psi_{\mathrm{h}}\left(x_{0}\right)-\psi_{\mathrm{h}}\left(x_{1}\right)<0
\end{aligned}
$$

The first limit in (2.15) follows since

$$
\lim _{a \rightarrow A-} x_{0}(a)=\lim _{a \rightarrow A-} x_{1}(a)=\bar{x}(A)
$$

by Proposition 2.1. The second limit is then a trivial consequence of (2.14) and the first limit.

\section{PROPOSITION 2.3}

For each $a \in(0, A)$ let $x_{0}(a)$ and $x_{1}(a)$ be the two solutions of $\psi_{a}^{\prime}(x)=\lambda$ given by Proposition 2.1. Then for each $0<c<L$, there exists a unique $a=a(c) \in(0, A)$ s.t. the triplet $\left(a(c), x_{0}(a(c)), x_{1}(a(c))\right)$ solves the system of equations

$$
\begin{aligned}
& \psi_{a}^{\prime}\left(x_{0}\right)=\lambda \\
& \psi_{a}^{\prime}\left(x_{1}\right)=\lambda \\
& \psi_{a}\left(x_{1}\right)=\psi_{a}\left(x_{0}\right)+c+\lambda\left(x_{1}-x_{0}\right)
\end{aligned}
$$

Moreover

$$
\lim _{c \rightarrow 0+} a(c)=A \quad \text { and } \quad \lim _{c \rightarrow 0+} x_{0}(a(c))=\lim _{c \rightarrow 0+} x_{1}(a(c))=\bar{x}(A)
$$

\section{PROOF}

The first two equations are satisfied for any $a \in(0, A)$, so we need only to consider the third equation. Note that by the definition of $g(a)$, this equation is equivalent to the statement

$$
g(a)=c
$$


Since $x_{0}(a)<x_{1}(a)$ and $\psi_{h}(x)$ is an increasing function, it follows from Lemma 2.2 that the function $a \mapsto g(a)$ is strictly decreasing from $L$ to 0 . Hence this equation has a unique solution $a=a(c)$ for any $0<c<L$. We put $x_{0}(c)=x_{0}(a(c))$ and $x_{1}(c)=x_{1}(a(c))$. As $c \rightarrow 0$, then $a(c) \rightarrow A$. Hence by Proposition 2.1, $\lim _{c \rightarrow 0+} x_{0}(a(c))=\lim _{c \rightarrow 0+} x_{1}(a(c))=\bar{x}(A)$.

Now for each $c>0$, let $\psi_{c}(x)=-a(c) \psi_{\mathrm{h}}(x)+\psi_{\mathrm{s}}(x)$ where $a=a(c)$ is the unique number given by Proposition 2.3. Then we can prove the following result

PROPOSITION 2.4

$$
\lim _{c \rightarrow 0+} \frac{d}{d c} \psi_{c}(x)=+\infty
$$

\section{PROOF}

We differentiate both sides of $g(a(c))=c$ w.r.t. $c$ to get

$$
\frac{d}{d c} a(c)=\frac{1}{\psi_{\mathrm{h}}\left(x_{0}(c)\right)-\psi_{\mathrm{h}}\left(x_{1}(c)\right)}
$$

Hence

$$
\lim _{c \rightarrow 0+} \frac{d}{d c} a(c)=-\infty
$$

and the proposition follows immediately from this.

For $0<c<L$, let $a^{*}=a(c), x_{0}^{*}=x_{0}\left(a^{*}\right)$ and $x_{1}^{*}=x_{1}\left(a^{*}\right)$. With

$$
\psi_{a^{*}}(x)=-a^{*} \psi_{\mathrm{h}}(x)+\psi_{\mathrm{s}}(x)
$$

define

$$
\phi_{c}(s, x)= \begin{cases}e^{-\rho s} \psi_{a^{*}}(x) & \text { for } x \leq x_{1}^{*} \\ e^{-\rho s} \psi_{a^{*}}\left(x_{0}^{*}\right)+c+\lambda e^{-\rho s}\left(x-x_{0}^{*}\right) & \text { for } x>x_{1}^{*}\end{cases}
$$

\section{THEOREM 2.5}

Assume that we can find functions $\psi_{h}$ and $\psi_{s}$ satisfying the conditions $\mathcal{A} 1-\mathcal{A} 6$, and assume that

$$
\begin{gathered}
\beta\left(x_{1}^{*}\right) \neq 0 \\
-\rho\left(\psi_{a^{*}}\left(x_{1}\right)+\lambda\left(x-x_{1}\right)\right)+\alpha(x) \lambda+x^{2} \geq 0 \quad \text { for all } x>x_{1}^{*} \\
e^{-\rho t}\left(\left|\psi_{\mathbf{s}}\left(X_{t}\right)\right|+\left|X_{t}\right|\right) \rightarrow 0 \text { as } t \rightarrow \infty \text { a.s. } Q^{(s, x)} \text { for all }(s, x) \in \mathbf{R}^{2}
\end{gathered}
$$

Then $\phi_{c}(s, x)=V_{c}(s, x)$ is the solution to (1.31), and the following impulse control is optimal 


$$
\begin{aligned}
& \tau_{0}^{*}=0 \\
& \tau_{k+1}^{*}=\inf \left\{t>\tau_{k}^{*} \mid X_{t}^{\nu^{*}} \geq x_{1}^{*}\right\}, k=0,1, \ldots \\
& \zeta_{0}^{*}= \begin{cases}x-x_{0}^{*} & \text { if } x \geq x_{1}^{*} \\
0 & \text { otherwise }\end{cases} \\
& \zeta_{k+1}^{*}=x_{1}^{*}-x_{0}^{*}
\end{aligned}
$$

Moreover

$$
\lim _{c \rightarrow 0+} \frac{d}{d c} \phi_{c}(s, x)=+\infty
$$

\section{PROOF}

Note that by construction, $\phi_{c}(s, x)$ satisfies (1.23)-(1.25) in Corollary 1.4. (1.26) and (1.27) are clear from the assumptions. To verify (1.28), we see that

$$
\Phi^{\prime \prime}\left(x_{0}^{*}\right)=-a^{*} \psi_{h}^{\prime \prime}\left(x_{0}^{*}\right)+\psi_{s}^{\prime \prime}\left(x_{0}^{*}\right)=\psi_{h}^{\prime \prime}\left(x_{0}^{*}\right)\left(\theta\left(x_{0}^{*}\right)-a^{*}\right)>0
$$

since $\theta$ is a decreasing function with $\theta\left(\bar{x}\left(a^{*}\right)\right)=a^{*}$ and $x_{0}^{*}<\bar{x}\left(a^{*}\right)$. Now if $x \geq x_{1}^{*}$, then $\psi_{\mathrm{s}}^{-}=0$, and if $x<x_{1}^{*}$ then the term $-a^{*} \phi_{\mathrm{h}}$ is uniformly bounded. By $(2.6) \phi_{\mathrm{s}}^{-}=0$, and (1.30) follows. To verify (1.31), note that $-a^{*} \psi_{\mathrm{h}}(x)$ is uniformly bounded when $x<x_{1}$ and that $\Phi(x)$ grows linearly outside this set. Since any admissible control gives a reduction in $\left|X_{t}\right|,(2.29)$ is sufficient for (1.31). Hence all the conditions in Corollary 1.4 are satisfied.

If $x \leq x_{1}^{*}$, (2.31) follows from Proposition 2.4. When $x>x_{1}^{*}$, then

$$
\phi_{c}(s, x)=e^{-\rho s}\left(-a^{*}(c) \psi_{\mathrm{h}}\left(x_{0}^{*}(c)\right)+\psi_{\mathrm{s}}\left(x_{0}^{*}(c)\right)+c+\lambda e^{-\rho s}\left(x-x_{0}^{*}(c)\right)\right)
$$

Hence

$$
\begin{aligned}
\frac{d}{d c} \phi_{c}(s, x) & =-e^{-\rho s} \psi_{\mathrm{h}}\left(x_{0}^{*}(c)\right) \frac{d}{d c} a^{*}(c) \\
& +1+e^{-\rho s}\left(\psi_{c}^{\prime}\left(x_{0}^{*}(c)\right)-\lambda\right) \frac{d}{d c} x_{0}^{*}(c) \\
& =1-e^{-\rho s} \psi_{\mathrm{h}}\left(x_{0}^{*}(c)\right) \frac{d}{d c} a^{*}(c)
\end{aligned}
$$

As $c \rightarrow 0^{+}$, then $x_{0}^{*}(c) \rightarrow \bar{x}(A)$, and (2.31) follows from this since $\frac{d}{d c} a^{*}(c) \rightarrow-\infty$ like in the proof of Proposition 2.4.

\section{Remarks}

In the examples we consider in Section $3, \phi_{\mathrm{S}}$ is a polynomial of order 2. In this case (2.29) follows from

$$
e^{-\rho t} X_{t}^{2} \rightarrow 0 \text { as } t \rightarrow \infty \text { a.s. } Q^{(s, x)} \text { for all }(s, x) \in \mathbf{R}^{2}
$$

To simplify the verification of $(2.28)$ we note that since $\psi_{a^{*}}^{\prime \prime}(x)<0$ when $x>x_{1}^{*}$, then by Taylors formula

$$
\int \psi_{a^{*}}\left(x_{1}^{*}+h\left(x_{1}^{*}\right) \gamma(y)\right)-\psi_{a^{*}}\left(x_{1}^{*}\right)-\psi_{a^{*}}^{\prime}\left(x_{1}^{*}\right) h\left(x_{1}^{*}\right) \gamma(y) m(d y)<0
$$


Hence using that $\psi_{a^{*}}$ satisfies $L_{0} \psi_{a^{*}}\left(x_{1}^{*}\right)+\left(x_{1}^{*}\right)^{2}=0$, we get

$$
\begin{aligned}
& -\rho\left(\psi_{a^{*}}\left(x_{1}^{*}\right)+\lambda\left(x-x_{1}^{*}\right)\right)+\alpha(x) \lambda+x^{2} \\
& =-\rho \psi_{a^{*}}\left(x_{1}^{*}\right)+\alpha\left(x_{1}\right) \psi_{a^{*}}^{\prime}\left(x_{1}^{*}\right)+\frac{1}{2} \beta\left(x_{1}\right)^{2} \psi_{a^{*}}^{\prime \prime}\left(x_{1}^{*}\right)+\left(x_{1}^{*}\right)^{2} \\
& +\int \psi_{a^{*}}\left(x_{1}^{*}+h\left(x_{1}^{*}\right) \gamma(y)\right)-\psi_{a^{*}}\left(x_{1}^{*}\right)-\psi_{a^{*}}^{\prime}\left(x_{1}^{*}\right) h\left(x_{1}^{*}\right) \gamma(y) m(d y) \\
& +\left(\alpha(x)-\alpha\left(x_{1}^{*}\right)\right) \lambda+\left(x^{2}-\left(x_{1}^{*}\right)^{2}\right)-\frac{1}{2} \beta\left(x_{1}\right)^{2} \psi_{a^{*}}^{\prime \prime}\left(x_{1}^{*}\right) \\
& -\int \psi_{a^{*}}\left(x_{1}^{*}+h\left(x_{1}^{*}\right) \gamma(y)\right)-\psi_{a^{*}}\left(x_{1}^{*}\right)-\psi_{a^{*}}^{\prime}\left(x_{1}^{*}\right) h\left(x_{1}^{*}\right) \gamma(y) m(d y) \\
& \geq\left(\alpha(x)-\alpha\left(x_{1}^{*}\right)\right) \lambda+\left(x^{2}-\left(x_{1}^{*}\right)^{2}\right)
\end{aligned}
$$

If in addition $\alpha(x)=\alpha \cdot x$, we see that for all $x>x_{1}^{*}$

$$
\begin{aligned}
& -\rho\left(\psi_{a^{*}}\left(x_{1}^{*}\right)+\lambda\left(x-x_{1}^{*}\right)+\alpha(x) \lambda+x^{2}\right. \\
& \geq\left(\alpha x-\alpha x_{1}^{*}\right) \lambda+\left(x^{2}-\left(x_{1}^{*}\right)^{2}\right) \\
& =\left(x-x_{1}^{*}\right)\left(x+x_{1}^{*}+\alpha \lambda\right) \geq\left(x-x_{1}^{*}\right)(2 \bar{x}(A)+\alpha \lambda)
\end{aligned}
$$

Hence if $\alpha(x)=\alpha \cdot x$, then (2.28) is OK if $\bar{x}(A) \geq-\frac{\alpha \lambda}{2}$.

From the calculation above it follows that if $\bar{x}(A)<-\frac{\alpha \lambda}{2}$ and $\gamma=0$, then (2.28) fails if $c$ is sufficiently small. Hence the condition above is necessary for this case.

3. Discussion of particular cases

\subsection{Brownian motion}

$$
d X_{t}=0 d t+1 d B_{t}
$$

In this case we have $x_{\text {lower }}=-\infty$ and $x_{\text {upper }}=+\infty$ and consider the differential equation

$$
\frac{1}{2} \psi^{\prime \prime}-\rho \psi+x^{2}=0
$$

It is easy to see that

$$
\psi_{\mathrm{h}}(x)=e^{\sqrt{2 \rho} x} \quad \psi_{\mathrm{s}}(x)=\frac{1}{\rho} x^{2}+\frac{1}{\rho^{2}}
$$

Properties $\mathcal{A} 1$ to $\mathcal{A} 5$ are obvious. As for $\mathcal{A} 6$, we get

$$
\theta(x)=\frac{1}{\rho^{2}} e^{-\sqrt{2 \rho} x}
$$

Hence $\theta^{-1}(a)=-\frac{1}{\sqrt{2 \rho}} \ln \left(\rho^{2} a\right)$, and (2.4) takes the form

$$
-A \sqrt{2 \rho} e^{\sqrt{2 \rho}\left(-\frac{1}{\sqrt{2 \rho}} \ln \left(\rho^{2} A\right)\right.}+\frac{2}{\rho}\left(-\frac{1}{\sqrt{2 \rho}} \ln \left(\rho^{2} A\right)\right)-\lambda=0
$$

This we can simplify to get

$$
A=\frac{1}{\rho^{2}} \exp \left(-1-\frac{\lambda \rho \sqrt{2 \rho}}{2}\right)
$$


and

$$
\bar{x}(A)=\frac{\rho \lambda}{2}+\frac{1}{\sqrt{2 \rho}}
$$

In this case we can prove that $L=+\infty$. First note that since $\psi_{\mathrm{s}}(x)=\psi_{\mathrm{s}}(x)=\frac{1}{\rho} x^{2}+\frac{1}{\rho^{2}}$, then $\psi_{\mathrm{s}}^{\prime}(0)=0$. Hence $f_{a}(0)<0$, so $x_{0}(a)>0$. Since $x_{0}^{\prime}(a)>0$, it will follow that $0 \leq x_{0}(a) \leq \bar{x}(A)$ for all $a \in(0, A)$. On the other hand $x_{1}(a)>\bar{x}(a) \rightarrow+\infty$ as $a \rightarrow 0+$. Now we can use that $\psi_{\mathrm{h}}^{\prime}(x)=\sqrt{2 \rho} \psi_{\mathrm{h}}(x)$ in (2.19) to show that

$$
g(a)=\frac{1}{\rho}\left(x_{1}(a)-x_{0}(a)\right)\left(x_{1}(a)+x_{0}(a)-2 \bar{x}(A)\right)
$$

Since all terms except $x_{1}(a)$ are uniformly bounded, it follows that $\lim _{a \rightarrow 0+} g(a)=L=+\infty$. Hence all the basic conditions $\mathcal{A} 1-\mathcal{A} 6$ are satisfied. Since $\beta=1$ the condition (2.27) is trivial. Using the remarks below Theorem 2.5, we see that since $\alpha=0$, then (2.28) is OK. Brownian motion clearly satisfies (2.33) which implies (2.29). Hence the conclusions in Theorem 2.5 follow for all $c>0$ in this case.

\subsection{Geometric Brownian motion with jumps}

$$
d X_{t}=\alpha X_{t} d t+\beta X_{t} d B_{t}+X_{t-} \int_{\mathbf{R}} \gamma(y) \tilde{N}(d t, d y) \quad \text { where } \gamma(y) \geq 0
$$

We assume that

$$
\rho> \begin{cases}2 \alpha+\beta^{2}+\int \gamma^{2}(y) m(d y) & \text { if } \alpha \geq 0 \\ \alpha+\beta^{2}+\int \gamma^{2}(y) m(d y) & \text { if } \alpha<0\end{cases}
$$

We always have $x_{\text {lower }}=0$ and $x_{\text {upper }}=+\infty$ and consider the differential equation

$$
\frac{1}{2} \beta^{2} x^{2} \psi^{\prime \prime}+\alpha x \psi^{\prime}+\int_{\mathbf{R}}\left(\psi(x+x \gamma(y))-\psi(x)-\psi^{\prime}(x) x \gamma(y)\right) m(d y)-\rho \psi+x^{2}=0
$$

Now assume that we have a special solution of the form $\psi_{h}(x)=C x^{2}$. When we insert this in (3.11), we get

$$
C \beta^{2} x^{2}+2 \alpha C x^{2}+C x^{2} \int_{\mathbf{R}}\left((1+\gamma(y))^{2}-1-2 \gamma(y)\right) m(d y)-\rho C x^{2}+x^{2}=0
$$

Hence, if $\rho>2 \alpha+\beta^{2}+\int_{\mathbf{R}} \gamma^{2}(y) m(d y)$, we find

$$
C=\frac{1}{\rho-2 \alpha-\beta^{2}-\int_{\mathbf{R}} \gamma^{2}(y) m(d y)}
$$

By Itôs formula,

$$
E\left[X_{t}^{2}\right]=x^{2}+\left(2 \alpha+\beta^{2}+\int_{\mathbf{R}} \gamma^{2}(y) m(d y)\right) \int_{0}^{t} E\left[X_{s}^{2}\right] d s
$$

which gives

$$
E\left[X_{t}^{2}\right]=x^{2} \exp \left[\left(2 \alpha+\beta^{2}+\int_{\mathbf{R}} \gamma^{2}(y) m(d y)\right) t\right]
$$


So

$$
J_{c}^{\emptyset}(s, x)= \begin{cases}+\infty & \text { if } \rho \leq 2 \alpha+\beta^{2}+\int_{\mathbf{R}} \gamma^{2}(y) m(d y) \\ e^{-\rho s} \frac{x^{2}}{\rho-2 \alpha-\beta^{2}-\int_{\mathbf{R}} \gamma^{2}(y) m(d y)} & \text { if } \rho>2 \alpha+\beta^{2}+\int_{\mathbf{R}} \gamma^{2}(y) m(d y)\end{cases}
$$

We only consider the case where $\rho>2 \alpha+\beta^{2}+\int_{\mathbf{R}} \gamma^{2}(y) m(d y)$. Next we show that there exists $\delta>2$ such that $\psi_{h}(x)=x^{\delta}$ is a solution of the corresponding homogeneous equation.

$$
\left.\frac{1}{2} \beta^{2} x^{2} \psi^{\prime \prime}+\alpha x \psi^{\prime}+\int_{\mathbf{R}}(\psi(x+x \gamma(y)))-\psi(x)-\psi^{\prime}(x) x \gamma(y)\right) m(d y)-\rho \psi=0
$$

If we insert $\psi(x)=x^{\delta}$ in (3.17), we obtain

$$
\frac{1}{2} \beta^{2} \delta(\delta-1) x^{\delta}+\alpha \delta x^{\delta}+x^{\delta} \int_{\mathbf{R}}\left((1+\gamma(y))^{\delta}-1-\delta \gamma(y)\right) m(d y)-\rho x^{\delta}=0
$$

Hence it suffices to find $\delta>2$ s.t.

$$
\Theta(\delta):=\frac{1}{2} \beta^{2} \delta(\delta-1)+\alpha \delta+\int_{\mathbf{R}}\left((1+\gamma(y))^{\delta}-1-\delta \gamma(y)\right) m(d y)-\rho=0
$$

Observe that if we let $\Phi:(-1, \infty) \rightarrow \mathbf{R}$ be given by

$$
\Phi(u)=(1+u)^{\delta}-1-\delta u
$$

then $\Phi(0)=0$ and if $\delta>1$, then also

$$
\Phi^{\prime}(u)=\delta(1+u)^{\delta-1}-1=\left\{\begin{array}{cc}
<0 & \text { if } u<0 \\
>0 & \text { if } u>0
\end{array}\right.
$$

It follows that we always have $\Phi(u) \geq 0$. Then observe that

$$
\begin{aligned}
\Theta(2) & =\beta^{2}+2 \alpha+\int_{\mathbf{R}}\left((1+\gamma(y))^{2}-1-\delta \gamma(y)\right) m(d y)-\rho \\
& =2 \alpha+\beta^{2}+\int_{\mathbf{R}} \gamma^{2}(y) m(d y)-\rho \\
& <0
\end{aligned}
$$

by our choice of $\rho$. Since $\Phi \geq 0$, it is trivial to see that $\lim _{\delta \rightarrow+\infty} \Theta(\delta)=+\infty$. Hence we can always find $\delta>2$ s.t. $\Theta(\delta)=0$, which is (3.19).

We remark that in the classical case, i.e., with no jumps, then $\delta$ is given by the explicit expression

$$
\delta=\frac{\beta^{2}-2 \alpha+\sqrt{\left(\beta^{2}-2 \alpha\right)^{2}+8 \beta^{2} \rho}}{2 \beta^{2}}>0
$$

Observe that if $\rho=2 \alpha+\beta^{2}$, then $\delta=2$, hence for all parameters s.t. $\rho>2 \alpha+\beta^{2}$, we have

$$
\delta>2
$$

We hence have produced the following candidates 


$$
\psi_{\mathrm{h}}(x)=x^{\delta} \quad \psi_{\mathbf{s}}(x)=\frac{x^{2}}{\rho-2 \alpha-\beta^{2}-\int_{\mathbf{R}} \gamma^{2}(y) m(d y)}
$$

Properties $\mathcal{A} 1$ to $\mathcal{A} 5$ are again obvious. As for $\mathcal{A} 6$, we this time get

$$
\theta(x)=\frac{2}{\left(\rho-2 \alpha-\beta^{2}-\int_{\mathbf{R}} \gamma^{2}(y) m(d y)\right) \delta(\delta-2)} x^{2-\delta}
$$

Hence $\theta^{-1}(a)=\left(\frac{2}{a\left(\rho-2 \alpha-\beta^{2}-\int_{\mathbf{R}} \gamma^{2}(y) m(d y)\right) \delta(\delta-2)}\right)^{\frac{1}{\delta-2}}$, and one can verify that

$$
\begin{gathered}
A=\frac{2}{\left(\rho-2 \alpha-\beta^{2}-\int_{\mathbf{R}} \gamma^{2}(y) m(d y)\right) \delta(\delta-1)} . \\
\left(\frac{2(\delta-2)}{\left(\rho-2 \alpha-\beta^{2}-\int_{\mathbf{R}} \gamma^{2}(y) m(d y)\right) \lambda(\delta-1)}\right)^{\delta-2}
\end{gathered}
$$

and

$$
\bar{x}(A)=\frac{\lambda(\delta-1)\left(\rho-2 \alpha-\beta^{2}-\int_{\mathbf{R}} \gamma^{2}(y) m(d y)\right)}{2(\delta-2)}
$$

Also in this case we can prove that $L=+\infty$. Here $\psi_{\mathbf{S}}(x)=\frac{x^{2}}{\rho-2 \alpha-\beta^{2}-\int_{\mathbf{R}} \gamma^{2}(y) m(d y)}$. In this case we clearly have $0 \leq x_{0}(a) \leq \bar{x}(A)$ for all $a \in(0, A)$, and also $\lim _{a \rightarrow 0+} x_{1}(a)=+\infty$. Now use $x \psi_{\mathrm{h}}^{\prime}(x)=\delta \psi_{\mathrm{h}}(x)$ in $(2.19)$ to show

$$
g(a)=\frac{\delta-2}{\delta\left(\rho-2 \alpha-\beta^{2}-\int_{\mathbf{R}} \gamma^{2}(y) m(d y)\right)}\left(x_{1}(a)-x_{0}(a)\right)\left(x_{1}(a)+x_{0}(a)-2 \bar{x}(A)\right)
$$

Then $\lim _{a \rightarrow 0+} g(a)=L=+\infty$. All the basic conditions $\mathcal{A} 1-\mathcal{A} 6$ are satisfied and (2.27) is trivial. Using the remarks below Theorem 2.5, we see that (2.28) is trivial if $\alpha \geq 0$. If $\alpha<0$, it follows easily from (3.10) and (3.28) that $\bar{x}(A)>-\frac{\alpha \lambda}{2}$ also in this case. Hence (2.28) follows. To verify (2.29), note that $X_{t}$ is given by the explicit expression

$$
X_{t}=X_{0} \exp \left[\left(\alpha-\frac{1}{2} \beta^{2}\right) t+\left(E\left[N_{t}\right]-K t\right)+\beta B_{t}+\left(N_{t}-E\left[N_{t}\right]\right)\right]
$$

where $N_{t}=\int_{0}^{t^{+}} \int_{\mathbf{R}} \ln [1+\gamma(y)] N(d s, d y)$ and $K=\int_{\mathbf{R}} \gamma(y) m(d y)$. Here both limits

$$
\frac{B_{t}}{t} \rightarrow 0 \quad \text { and } \quad \frac{N_{t}-E\left[N_{t}\right]}{t} \rightarrow 0 \quad \text { a.s. as } t \rightarrow \infty
$$

We can also see that

$$
\begin{aligned}
E\left[N_{t}\right] & =\int_{0}^{t} \int_{\mathbf{R}} \ln [1+\gamma(y)] m(d y) d s=t \int_{\mathbf{R}} \ln [1+\gamma(y)] m(d y) \\
& \leq t \ln \left[\int_{\mathbf{R}} 1+\gamma(y) m(d y)\right]=t \ln [1+K] \leq K t
\end{aligned}
$$

Hence $E\left[N_{t}\right]-K t \leq 0$. Now (2.29) follows easily from (2.33), (3.19), (3.30) and (3.31). Again all the conditions in Theorem 2.5 are satisfied, and the conclusions in Theorem 2.5 follow for all $c>0$. 


\subsection{The Ornstein-Uhlenbeck process}

$$
d X_{t}=-\alpha X_{t} d t+\beta d B_{t}
$$

where $\alpha, \beta, \rho>0$. In this case we have $x_{\text {lower }}=-\infty$ and $x_{\text {upper }}=+\infty$ and consider the differential equation

$$
\frac{1}{2} \beta^{2} \psi^{\prime \prime}-\alpha x \psi^{\prime}-\rho \psi+x^{2}=0
$$

It is straightforward to find $\psi_{\text {s }}$, and we get

$$
\psi_{\mathbf{s}}(x)=\frac{1}{\rho+2 \alpha} x^{2}+\frac{\beta^{2}}{\rho(\rho+2 \alpha)}
$$

To find a homogeneous solution, i.e., to solve

$$
\frac{1}{2} \beta^{2} \psi^{\prime \prime}-\alpha x \psi^{\prime}-\rho \psi=0
$$

is, however, more complicated. It is well known that solutions of (3.35) can be expressed in terms of Kummers function $M(a, b, x)$. This function is defined through the expression

$$
M(a, b, x)=1+\frac{a}{b} x+\frac{a(a+1)}{b(b+1) 2 !} x^{2}+\cdots+\frac{a(a+1) \cdots(a+n-1)}{b(b+1) \cdots(b+n-1) n !} x^{n}+\cdots
$$

We will need the following properties of $M(a, b, x)$, see [AS]

$\mathcal{K} 1: w(x)=M(a, b, x)$ is a solution to

$$
x w^{\prime \prime}+(b-x) w^{\prime}-a w=0
$$

$\mathcal{K} 2: M^{\prime}(a, b, x)=\frac{a}{b} M(a+1, b+1, x)$

$\mathcal{K} 3:$ As $x \rightarrow+\infty$

$$
M(a, b, x)=\frac{\Gamma(b)}{\Gamma(a)} e^{x} x^{a-b}\left(1+O\left(|x|^{-1}\right)\right)
$$

$\mathcal{K} 4$ :

$$
x M(a, b+1, x)=b M(a, b, x)-b M(a-1, b, x)
$$

$\mathcal{K} 5$ :

$$
a M(a+1, b, x)-(1+a-b) M(a, b, x)=(b-1) M(a, b+1, z)
$$

Using the above properties, we can prove the following proposition. 


\section{PROPOSITION 3.1}

If we put $a=\frac{\rho}{2 \alpha}, k=\frac{\alpha}{\beta^{2}}$, then

$$
\psi_{h}(x)=M\left(a, \frac{1}{2}, k x^{2}\right)+\frac{2 \sqrt{\alpha} \Gamma\left[a+\frac{1}{2}\right]}{\beta \Gamma[a]} x M\left(a+\frac{1}{2}, \frac{3}{2}, k x^{2}\right)
$$

is a solution to (3.35) satisfying all the conditions in Theorem 2.5.

\section{PROOF}

It is well known, and in fact straightforward to verify (using $\mathcal{K} 1$ ) that

$$
y_{1}=M\left(a, \frac{1}{2}, k x^{2}\right) \quad \text { and } \quad y_{2}=x M\left(a+\frac{1}{2}, \frac{3}{2}, k x^{2}\right)
$$

are two linearly independent solutions to (3.35). From general theory, see, e.g., [BS], we know that there exist a solution $y_{h}$ of (3.35) which satisfies $\mathcal{A} 1-\mathcal{A} 3$ when $n=0$ in these statements. Clearly $y_{h}$ can be expressed on the form

$$
y_{h}=C_{1} y_{1}+C_{2} y_{2}
$$

and such a function $y_{h}$ is unique up to a (positive) multiplicative constant. Without loss of generality we can then assume that $C_{1}=1$. Using the property $\mathcal{K} 3$, we can see that we have $\lim _{x \rightarrow-\infty} y_{h}(x)=0$ only if $C_{2}=\frac{2 \sqrt{\alpha} \Gamma\left[a+\frac{1}{2}\right]}{\beta \Gamma[a]}$. This proves that the function given by $(3.41)$ satisfies $\mathcal{A} 1-\mathcal{A} 3$ when $n=0$ in these statements.

We now compute $\psi_{h}^{\prime}$ using $\mathcal{K} 2, \mathcal{K} 4$, and $\mathcal{K} 5$ to rewrite the expression.

$$
\begin{aligned}
\psi_{h}^{\prime} & =4 a k x K\left(a+1, \frac{3}{2}, k x^{2}\right)+C_{2} K\left(a+\frac{1}{2}, \frac{3}{2}, k x^{2}\right) \\
& +C_{2} \frac{2}{3}(1+2 a) k x^{2} K\left(a+\frac{3}{2}, \frac{5}{2}, k x^{2}\right) \\
& =4 a k x K\left(a+1, \frac{3}{2}, k x^{2}\right)+C_{2} K\left(a+\frac{1}{2}, \frac{3}{2}, k x^{2}\right) \\
& +C_{2}(1+2 a) K\left(a+\frac{3}{2}, \frac{3}{2}, k x^{2}\right)-C_{2}(1+2 a) K\left(a+\frac{1}{2}, \frac{3}{2}, k x^{2}\right) \\
& =4 a k x K\left(a+1, \frac{3}{2}, k x^{2}\right) \\
& +2 C_{2}\left(\left(a+\frac{1}{2}\right) M\left(a+\frac{3}{2}, \frac{3}{2}, k x^{2}\right)-a M\left(a+\frac{1}{2}, \frac{3}{2}, k x^{2}\right)\right) \\
& =4 a k x K\left(a+1, \frac{3}{2}, k x^{2}\right)+C_{2} K\left(a+\frac{1}{2}, \frac{1}{2}, k x^{2}\right) \\
& =C_{2}\left(K\left(a+\frac{1}{2}, \frac{1}{2}, k x^{2}\right)+\frac{4 a k}{C_{2}} x K\left(a+1, \frac{3}{2}, k x^{2}\right)\right) \\
& =C_{2}\left(K\left(a+\frac{1}{2}, \frac{1}{2}, k x^{2}\right)+\frac{2 \sqrt{\alpha} \Gamma[a+1]}{\beta \Gamma\left[a+\frac{1}{2}\right]} x K\left(a+1, \frac{3}{2}, k x^{2}\right)\right)
\end{aligned}
$$

If we inspect the expression in the brackets, this is similar to the expression (3.41), the only difference being that $a$ is replaced by $a+\frac{1}{2}$. Since we in fact have proved that $\mathcal{A} 1-\mathcal{A} 3, n=0$ are OK for all such expressions, $\psi_{h}^{\prime}$ also satisfies $\mathcal{A} 1-\mathcal{A} 3, n=0$. Hence we have proved that $\mathcal{A} 1-\mathcal{A} 3$ are OK when $n=0,1$. Repeating this argument, is follows that that the statements in $\mathcal{A} 1-\mathcal{A} 3$ are satisfied for all $n \in \mathbf{N}$. 
Since $\psi_{\mathrm{s}}(x)=C_{3} x^{2}+C_{4}$, the properties $\mathcal{A} 4$ and $\left.\mathcal{A} 6 \mathrm{a}, \mathrm{b}, \mathrm{c}\right)$ are immediate consequences of $\mathcal{A} 1-\mathcal{A} 3$. Since $\lim _{x \rightarrow+\infty} \psi_{s}(x)=\infty, \mathcal{A} 5$ follows from $\mathcal{A} 6 \mathrm{~b}$ ) by L'Hôpital's rule. We proceed to verify $\mathcal{A} 6 \mathrm{~d})$. To this end, we note that since $\bar{x}(a) \geq 0$

$$
0 \leq \alpha \bar{x}(a) \psi_{\mathrm{h}}^{\prime}(\bar{x}(a))=\frac{1}{2} \beta^{2} \psi_{\mathrm{h}}^{\prime \prime}(\bar{x}(a))-\rho \psi_{\mathrm{h}}(\bar{x}(a)) \leq \frac{1}{2} \beta^{2} \psi_{\mathrm{h}}^{\prime \prime}(\bar{x}(a))
$$

We now use this together with the relation

$$
\frac{\psi_{s}^{\prime \prime}(\bar{x}(a))}{\psi_{h}^{\prime \prime}(\bar{x}(a))}=a
$$

to see that

$$
a \psi_{h}^{\prime}(\bar{x}(a)) \leq a \frac{\beta^{2} \psi_{s}^{\prime \prime}(\bar{x}(a))}{2 \alpha \bar{x}(a) a}=\frac{\beta^{2} \psi_{s}^{\prime \prime}(\bar{x}(a))}{2 \alpha \bar{x}(a)}
$$

Since $\lim _{a \rightarrow 0+} \psi_{\mathrm{s}}^{\prime}(\bar{x}(a))=+\infty$, it follows that

$$
\lim _{a \rightarrow 0+}-a \psi_{h}^{\prime}(\bar{x}(a))+\psi_{\mathbf{s}}^{\prime}(\bar{x}(a))-\lambda=+\infty
$$

On the other hand it follows from $\mathcal{A} 6 \mathrm{a}, \mathrm{b}, \mathrm{c})$ that $\lim _{a \rightarrow+\infty} \bar{x}(a)=-\infty$. Hence from $\mathcal{A} 5$

$$
\lim _{a \rightarrow+\infty}-a \psi_{h}^{\prime}(\bar{x}(a))+\psi_{\mathbf{s}}^{\prime}(\bar{x}(a))-\lambda=-\infty
$$

Then from (3.47) and (3.48) we can finally conclude that there exist $A>0$ s.t.

$$
-A \psi_{h}^{\prime}(\bar{x}(A))+\psi_{\mathrm{s}}^{\prime}(\bar{x}(A))-\lambda=0
$$

which is $\mathcal{A} 6 \mathrm{~d})$. (2.27) is trivial. To verify (2.28), note that from (3.49) we get

$$
\psi_{\mathrm{s}}^{\prime}(\bar{x}(A))=\lambda+A \psi_{h}^{\prime}(\bar{x}(A)) \geq \lambda
$$

Using (3.31), we get

$$
\frac{2 \bar{x}(A)}{\rho+2 \alpha} \geq \lambda \Rightarrow \bar{x}(A) \geq \frac{\lambda}{2}(\rho+2 \alpha) \geq \frac{\lambda \alpha}{2}
$$

Hence by the remarks following Theorem 2.5 again, (2.28) is satisfied for all $\alpha \geq 0$ (Note that we have changed the sign of $\alpha$ in this case, so $\alpha$ is always negative according to the standard setup). (2.33) is clearly satisfied in this case. This implies (2.29) and so Theorem 2.5 also applies to this situation.

\section{ACKNOWLEDGEMENTS}

This work is supported by VISTA, a research cooperation between The Norwegian Academy of Science and Letters and Den Norske Stats Oljeselskap (Statoil). 


\section{REFERENCES}

[AS] M. Abramowitz and I. A. Stegun: Handbook of Mathematical Functions, Dover 1965.

[BL] A. Bensoussan and J. L. Lions: Impulse Control and Quasi-variational inequalities, Gauthier-Villars 1984.

[BS] A. N. Borodin and P. Salminen: Handbook of Brownian Motion: Facts and Formulae, Birhäuser 1996.

[BØ1] K. A. Brekke and B. Øksendal: The high contact principle as a sufficiency condition for optimal stopping. In D. Lund and B. Øksendal (editors): Stochastic Models and Option Values. North-Holland 1991, pp. 187-208.

[BØ2] K. A. Brekke and B. Øksendal: A verification theorem for combined stochastic control and impulse control. In L. Decreusefond, J. Gjerde, B. Øksendal and S. Ustunel (editors): Stochastic Analysis and Related Topics, Vol. 6. Birkhäuser 1998, pp. 211-220.

[F] N. C. Framstad, Combined Stochastic Control for Jump Diffusions with Applications to Economics, Cand. Scient. Thesis, University of Oslo 1997.

[HST] J. M. Harrison, T. M. Sellke and A. J. Taylor: Impulse control of Brownian motion. Mathematics of Operations Research 8 (1983), 454-466.

[IW] N. Ikeda and S. Watanabe: Stochastic Differential Equations and Diffusion Processes, 2 edn., North-Holland/Kodansha 1989.

[JS] M. Jeanblanc-Picqué and A. N. Shiryaev: Optimization of the flow of dividends. Russian Math. Surveys 50 (1995), 257-277.

[LØ] E. Lungu and B. Øksendal: Optimal harvesting from a population in a stochastic crowded environment. Preprint, University of Oslo 1997 (to appear in Mathematical Biosciences).

[MØ] G. Mundaca and B. Øksendal: Optimal stochastic intervention control with application to the exchange rate. Preprint, University of Oslo 1997 (to appear in J. Mathematical Economics).

[MR1] J. L. Menaldi and M. Robin: On some cheap control problems for diffusion processes. Transactions of the AMS, 278 (1983), 771-802.

[MR2] J. L. Menaldi and M. Robin: On singular stochastic control problems for diffusions with jumps. IEEE Transactions of Automatic Control, AC-29 (1984), 991-1004.

[Ø1] B. Øksendal: Stochastic Differential Equations, 5 edn., Springer Verlag 1998.

[Ø2] B. Øksendal: Stochastic control problems where small intervention costs have dramatic effects, manuscript, 1997.

[P] P. Protter: Stochastic Integration and Differential Equations. A New Approach. Springer-Verlag 1990.

[V] H. Varner: Some Impulse Control Problems with Applications to Economics. Cand. Scient. Thesis, University of Oslo 1997. 